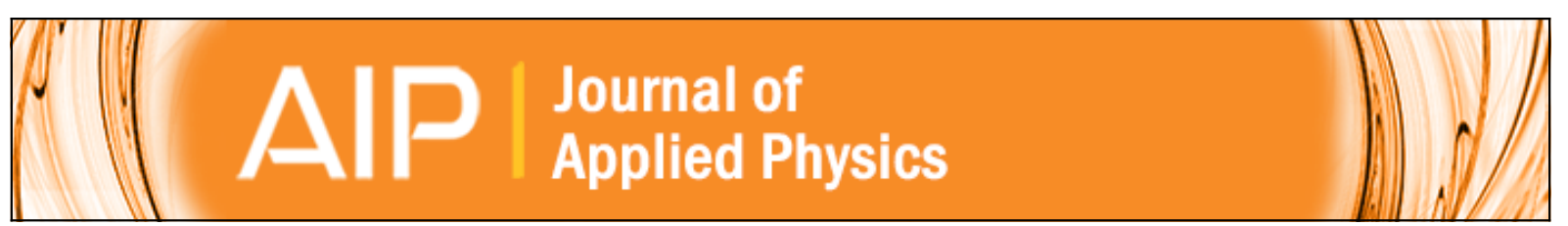

Comparison of light scattering in solar cells modeled by rigorous and scalar approach M. Ermes, K. Bittkau, and R. Carius

Citation: Journal of Applied Physics 113, 073104 (2013); doi: 10.1063/1.4790360

View online: http://dx.doi.org/10.1063/1.4790360

View Table of Contents: http://scitation.aip.org/content/aip/journal/jap/113/7?ver=pdfcov

Published by the AIP Publishing

$\stackrel{A}{A} \mathbb{P} P$ Re-register for Table of Content Alerts

Create a profile.

Sign up today! 


\title{
Comparison of light scattering in solar cells modeled by rigorous and scalar approach
}

\author{
M. Ermes, ${ }^{\text {a) }}$ K. Bittkau, and R. Carius \\ IEK5-Photovoltaik, Forschungszentrum Jülich GmbH, 52425 Jülich, Germany
}

(Received 31 July 2012; accepted 21 January 2013; published online 20 February 2013)

\begin{abstract}
We investigate and compare two very different approaches for obtaining light scattering properties at a rough interface with a focus on applications in silicon based thin-film solar cells. The scattering properties of a rigorous solving of Maxwell's equations and a recently developed model by Dominé et al. [J. Appl. Phys. 107, 044505 (2010)] based on a scalar approach are compared. The former yields extensive information and is very versatile, but needs much computation time. The latter is only applicable for single textures between two half-spaces and yields only scattering properties of said textures, but is extremely fast. The applicability and limitations of the scalar approach are examined. The results show that a combination of both can improve and accelerate simulation and development of new devices. (C) 2013 American Institute of Physics. [http://dx.doi.org/10.1063/1.4790360]
\end{abstract}

\section{INTRODUCTION}

In this article, light scattering in silicon based thin-film solar cells is discussed. These thin-film solar cells consist of hydrogenated amorphous (a-Si:H) and/or micro-crystalline silicon $(\mu \mathrm{c}-\mathrm{Si}: \mathrm{H})$ absorber layers with thicknesses between $300 \mathrm{~nm}(\mathrm{a}-\mathrm{Si}: \mathrm{H})$ and $2000 \mathrm{~nm}(\mu \mathrm{c}-\mathrm{Si}: \mathrm{H})$. These thicknesses are chosen based on the effects of light induced degradation of efficiency and the absorption coefficient of the material.

These thicknesses present a large challenge, since the absorption length of the incident light is much longer than the film thickness for wavelengths larger than $500 \mathrm{~nm}$. To extend the path length of the incident light, several approaches can be considered. ${ }^{1-5}$

One of the most promising is using textured transparent conductive oxides (TCO) as front contacts to scatter the incident light. In a multi-layered solar cell structure, each interface exhibits a texture that leads to light scattering, further prolonging the path of the incident light. These effects can also be investigated with a simplified system consisting of a single textured interface between two materials.

In our case, we focus on a textured aluminium-doped zinc oxide ( $\mathrm{ZnO}: \mathrm{Al})$ obtained by sputtering followed by wetchemical etching. ${ }^{1}$ The resulting texture is a crater-like structure with statistically distributed sizes, whereas longer etching times result in larger craters. The positive effect of such structures on the performance of silicon-based thin-film solar cells has been discussed in earlier works. ${ }^{6-14}$ Additionally, we investigate the scattering of Boron-doped zinc oxide (ZnO:B), obtained by liquid phase chemical vapor deposition (LPCVD), so we can cover a wider array of surface morphologies.

The scattering at such interfaces is commonly characterized by two quantities: Haze and angular intensity distribution (AID).

\footnotetext{
${ }^{a)}$ Electronic mail: m.ermes@fz-juelich.de.
}

The first describes the intensity of diffusely transmitted light-i.e., at an angle $>0^{\circ}$-normalized by the total transmitted intensity:

$$
H_{T}=T_{\text {diff }} / T_{\text {tot }} .
$$

The latter gives further information how much intensity was scattered into certain angles.

There have been some approaches to estimate these quantities for a given texture, such as the scalar scattering theory, ${ }^{15,16}$ which takes into account the rms roughness of a texture and a wavelength-dependent correcting function. Several modifications have been discussed to modify this approach for different textures. ${ }^{16}$ This approach leaves much to be desired, especially since light scattering at a $\mathrm{ZnO}: \mathrm{Al} /$ $\mu \mathrm{c}-\mathrm{Si}: \mathrm{H}$ interface is not easily accessible by experiment.

We chose to compare a new model proposed by Dominé et al. ${ }^{17}$ which has also been incorporated into other simulation tools, ${ }^{18}$ referred to as the phase model (PM), with a rigorous approach by finite-difference time-domain (FDTD) simulations, ${ }^{19}$ because the $\mathrm{PM}$ also shows a good agreement for scattering into silicon. ${ }^{20}$ Previous works in this field included estimations of the resulting absorption in a thin-film solar cell at single, representative wavelengths. ${ }^{21}$

The phase model takes into consideration a texture of an interface and the refractive indices of the adjacent materials, while neglecting absorption in both materials. From these quantities, a phase shift is calculated which depends on the path length in each material due to the different propagation speeds in different materials. It results in a complex phase for each point of the texture, explained in Eq. (3), as developed by Dominé. ${ }^{17}$

$$
\begin{aligned}
\phi_{\text {out }} & =\phi_{0}+\frac{2 \pi}{\lambda} n_{1} \cdot \zeta(x, y)+\frac{2 \pi}{\lambda} n_{2} \cdot(d-\zeta(x, y)) \\
& =\text { const }+\frac{2 \pi}{\lambda} \zeta(x, y)\left(n_{1}-n_{2}\right),
\end{aligned}
$$

with $\phi_{\text {out }}$ being the resulting phase, $\phi_{0}$ the initial phase, $\lambda$ the wavelength of the incident light in vacuum, and $n_{1}$ and $n_{2}$ are the refractive indices of the materials on the incident and 
transmitted side of the interface, respectively. $\zeta(x, y)$ represents the height of the texture at a given point $x$ and $y$ for a twodimensional scan.

Since this calculation is done for each point of the surface independently of all others, the result at each point only dependent on the height of the topography. As such, the phase-model is a quasi one-dimensional model. From the resulting pupil function, haze and AID can be calculated via fast Fourier transformation (FFT). The computing power necessary for these calculations is low enough to be used on even low-end workstations in very short time.

FDTD simulations, in contrast, need large amounts of CPU time and memory, resulting in a long computation time even on high-performance clusters, since the system must be modeled as a three-dimensional grid, with the domain-size and resolution being the most important factors. The former must be large enough so the results are not dominated by edge effects, while the latter must be high enough to resolve the features of the investigated textures.

These quantities result in a certain number of points to be simulated, with the total computation time scaling roughly linear with the number of points in the domain.

The advantage of time consuming FDTD simulations over the PM is its ability to model systems consisting of many different layers of various thicknesses and different optical properties, while the PM is limited to a single interface between non-absorbing materials. Therefore, for thin-film solar cell applications, FDTD simulations have to be considered.

In this article, we compare haze, AID and near-field intensities of different structures and different material combinations as obtained by PM and FDTD. If the light scattering properties resulting from PM and FDTD show a good enough agreement, the PM can be used to estimate the necessity of a rigorous FDTD simulation for a (multi-layer) system containing the analyzed interface.

\section{SAMPLE AND CALCULATION DETAILS}

For the comparison, different $\mathrm{ZnO}$ : $\mathrm{Al}$ layers were deposited on glass substrate by magnetron sputtering. The surface texture of four samples is achieved by chemical etching in 0.5 wt. \% hydrochloric acid. A fifth sample of $\mathrm{ZnO}: \mathrm{B}$, manufactured by LPCVD, which exhibits an as-grown roughness, was also investigated. This sample shows smaller, pyramidlike structures. It is investigated because of the very different type of surface morphology. The good performance of this type of structure in thin-film solar cells has been shown by Battaglia et $a .^{22}$ The five systems we investigated were

- A 30s etched $\mathrm{ZnO}: \mathrm{Al}$ structure with a $\mathrm{ZnO}: \mathrm{Al} /$ air interface ("small feature sample").

- An artificial structure created by stretching the height of a 40s etched $\mathrm{ZnO}: \mathrm{Al}$ surface by a factor of 3 to obtain steep flanks ("stretched sample").

- A 50s etched $\mathrm{ZnO}: \mathrm{Al}$ structure with a $\mathrm{ZnO}: \mathrm{Al} /$ air interface ("large feature air sample").

- The same texture with a $\mathrm{ZnO}: \mathrm{Al} / \mu \mathrm{c}-\mathrm{Si}: \mathrm{H}$ interface ("large feature $\mu \mathrm{c}-\mathrm{Si}: \mathrm{H}$ sample").

- A ZnO:B structure obtained by LPCVD ("LPCVD sample").

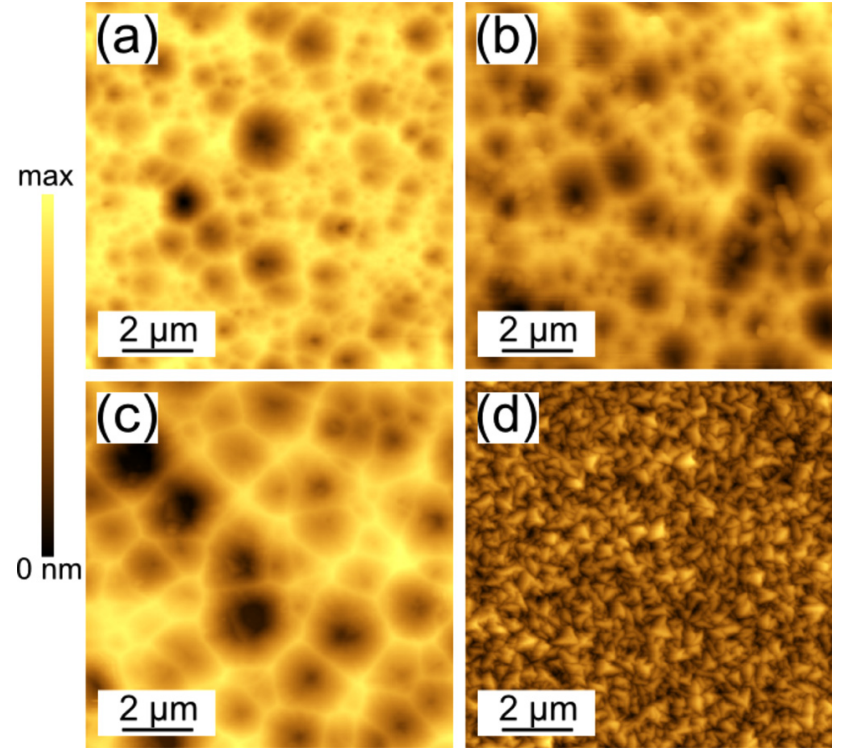

FIG. 1. Topographies of small feature sample (a) with heights up to $600 \mathrm{~nm}$, stretched sample (b, heights of up to $3100 \mathrm{~nm}$ ), and large feature air and $\mu \mathrm{c}$-Si:H sample (c) (heights up to $890 \mathrm{~nm}$ ) as measured by AFM. Panel (d) shows the surface of the LPCVD-grown $\mathrm{ZnO}$ :B layer (heights up to $540 \mathrm{~nm})$.

The topographies of these samples are shown in Fig. 1. Figure 2 shows the system as used for the models.

We assume that our investigated structures, with their combination of different textures (small feature, large feature air, and stretched sample as well as LPCVD sample) and materials (large feature $\mu \mathrm{c}-\mathrm{Si}: \mathrm{H}$ sample), cover a wide enough set of parameters important for light scattering at an interface to also have predictive power for LPCVD-grown $\mathrm{ZnO}$.

For both models, the same surface profile from atomic force microscopy (AFM) is taken as the input. The FDTD simulation was modeled with the $y z$-plane equivalent to the (flat) substrate surface. The texture was applied to a $\mathrm{ZnO}: \mathrm{Al}$ layer on top of that surface. The incident light was modeled as a plane wave propagating in $x$-direction, linearly polarized in $z$-direction. The rigorous solution of Maxwell's equations by means of FDTD simulation used periodic boundary conditions. The software used for FDTD simulations is a custommade interface to the MEEP FDTD solver, developed at MIT, ${ }^{23}$ optimized to handle textured interfaces. An additional difference between our FDTD and PM calculations is that in $\mathrm{PM}$, no material can be assumed as absorbing, while in FDTD, our $\mathrm{ZnO}: \mathrm{Al}$ layer is modeled from optical data



FIG. 2. Sketch of the system used in FDTD simulations. The dashed and dotted lines show the planes used for calculation of the AID (dashed: PM, dotted: FDTD) and light scattered into evanescent modes (dashed line). 


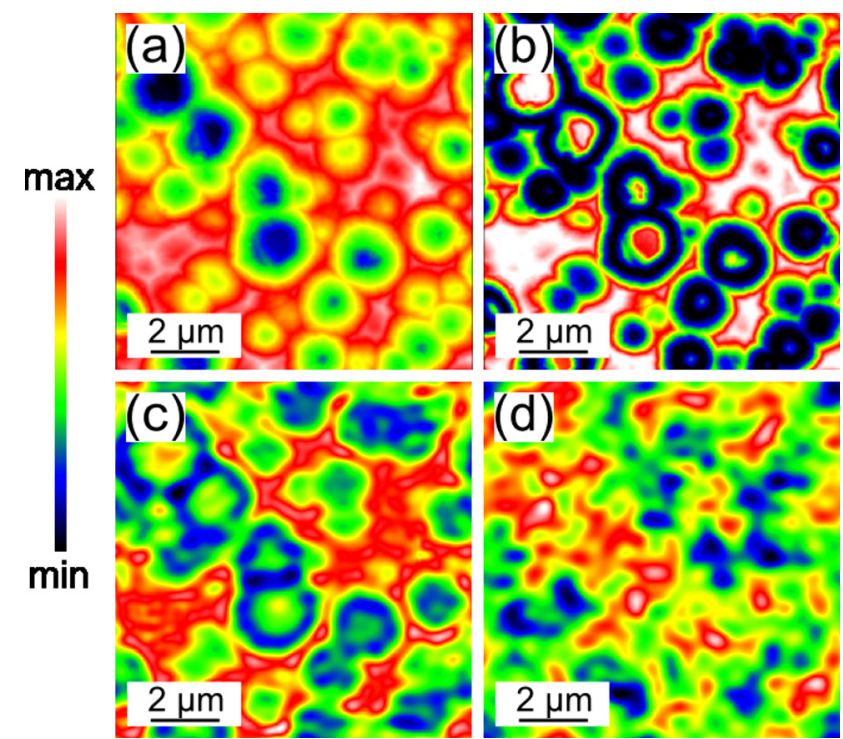

FIG. 3. Topography, PM and FDTD results for large feature air sample: Original topography (a), real part of the pupil function as obtained by PM (b), real part of the electric field in polarization direction obtained by FDTD above the highest peak of the topography (c), and $3.5 \mu \mathrm{m}$ above the surface (d). All calculations were performed at a wavelength of $600 \mathrm{~nm}$.

obtained by experiment. This leads to an additional investigation of the influence of absorption on the light scattering. For the $\mathrm{ZnO}: \mathrm{B}$ layer, the same optical data are used as for $\mathrm{ZnO}: \mathrm{Al}$ to investigate only the influence of different textures. In our case, PM and FDTD were used to calculate the phase and the electric field created by the topography, respectively (shown in Fig. 3). These complex functions are then used to calculate wavelength-dependent haze as well as angular intensity distribution and light scattered into evanescent modes.

The pupil function obtained by the PM is shown in the $y z$-plane at the absolute maximum of the texture, due to the nature of this model (see Fig. 2). Because the PM is used to ascertain the far field scattering of a texture, the results of the haze and AID calculations were compared to FDTD simulations at a longer distance from the sample of about $3.5 \mu \mathrm{m}$, whereas light scattered into evanescent modes were calculated from the $y z$-plane at the absolute maximum of the texture for both models.

Figure 3(a) shows the AFM image of a 50s etched $\mathrm{ZnO}: \mathrm{Al}$ surface ("large feature samples"). It exhibits craterlike structures of randomly distributed sizes. Figure 3(b) displays the real part of the pupil function obtained by PM for the large feature air sample. The real part of the electric field in a plane directly above the surface and $3.5 \mu \mathrm{m}$ above said surface are shown in Figs. 3(c) and 3(d), respectively. Both, pupil function of PM and electric field directly above the surface, reflect the shape of the topography. However, the onedimensional nature of the PM shows finer features at the crater rims, whereas the FDTD simulation shows much coarser structures because of the influence of all three dimensions.

\section{HAZE}

The haze of all samples was calculated for wavelengths between $300 \mathrm{~nm}$ and $1300 \mathrm{~nm}$ with steps of $50 \mathrm{~nm}$. Figures 4(a) and 4(b) show the results from both models for the small
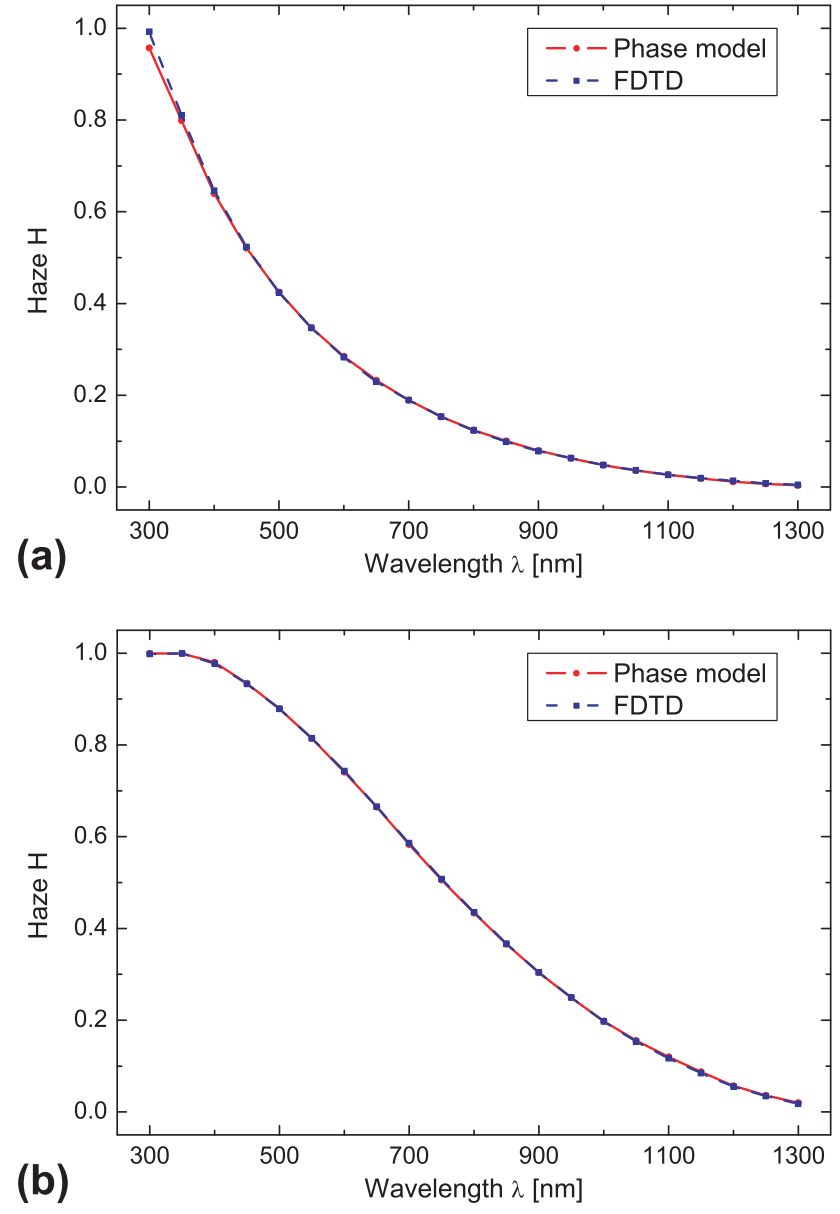

FIG. 4. Spectral haze of the small feature (a) and large feature air (b) sample calculated from PM and FDTD for a $\mathrm{ZnO}: \mathrm{Al} /$ air interface.

feature and large feature air sample, respectively. While for both samples a nearly perfect agreement is found, there is a slight difference for the small feature sample at $300 \mathrm{~nm}$ which results from numerical errors in the FDTD simulation; due to high absorption in the $\mathrm{ZnO}$ :Al layer at $300 \mathrm{~nm}$, the transmitted light intensity is very small. So small, in fact, that it is affected by the numerical accuracy of the simulation. The quantities used to calculate the haze are therefore numerical artifacts, which lead to a difference in haze. In the PM, in contrast to FDTD simulations, all materials are nonabsorbing, so a haze can be calculated.

Figures 5(a) and 5(b) show the spectral haze calculated for a $\mathrm{ZnO}: \mathrm{Al} / \mu \mathrm{c}-\mathrm{Si}: \mathrm{H}$ interface (large feature $\mu \mathrm{c}-\mathrm{Si}: \mathrm{H}$ sample) and a $\mathrm{ZnO}: \mathrm{Al} /$ air interface above an artificial steep-flank topography (stretched sample), respectively. Again, the haze shows good agreement for the most part of the spectrum. However, there is a small but clearly visible difference between PM and FDTD between $700 \mathrm{~nm}$ and $1250 \mathrm{~nm}$ for the large feature $\mu \mathrm{c}-\mathrm{Si}: \mathrm{H}$ sample and between $850 \mathrm{~nm}$ and $1200 \mathrm{~nm}$ for the stretched sample, resulting in a higher haze in the PM.

The LPCVD sample (Fig. 6) shows a very good agreement between FDTD and PM, with a visible deviation between $400 \mathrm{~nm}$ and $800 \mathrm{~nm}$. The largest difference is visible at $600 \mathrm{~nm}$.

Overall, it is found that the higher the refractive index difference and the steeper the flanks of the texture, the stronger the deviations of PM and FDTD. 

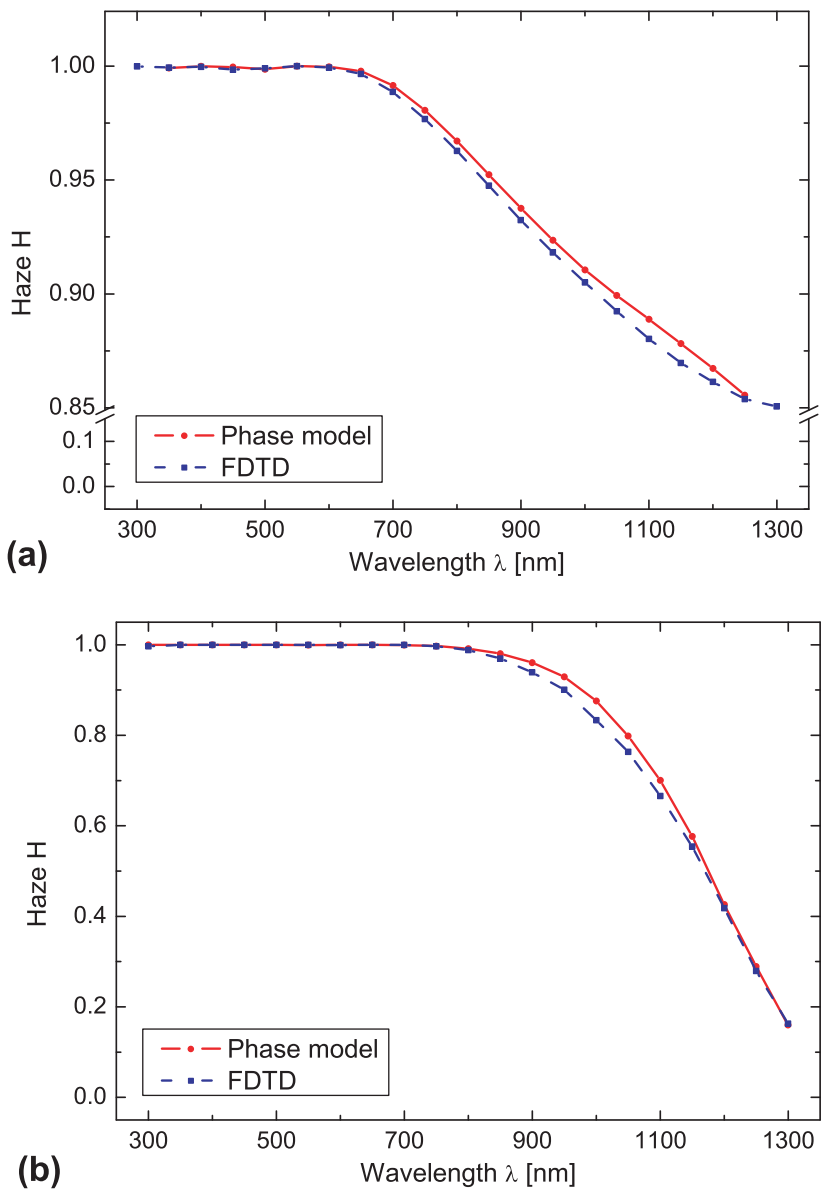

FIG. 5. Spectral haze of the large feature $\mu \mathrm{c}-\mathrm{Si}: \mathrm{H}$ (a) and stretched (b) sample.

A deeper investigation of this difference will be discussed in the next section by looking at the AID. Since the haze is calculated by the integrated intensity scattered at angles above $0^{\circ}$ (diffuse) and the intensity scattered into $0^{\circ}$ (specular), changes in the AID, especially at $0^{\circ}$, influence the haze.

\section{ANGULAR INTENSITY DISTRIBUTION}

In this section, the AID of all samples will be discussed for wavelengths of $600 \mathrm{~nm}$ and $1000 \mathrm{~nm}$ calculated with both

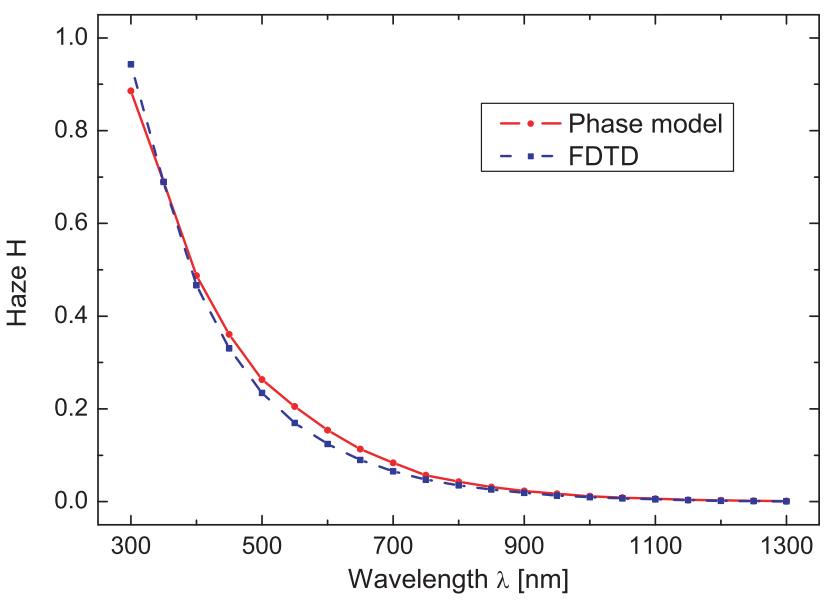

FIG. 6. Spectral haze of the LPCVD sample. models. The calculations at $600 \mathrm{~nm}$ show a good agreement of haze values for all samples, while at $1000 \mathrm{~nm}$ the strongest deviation of the large feature $\mu \mathrm{c}-\mathrm{Si}: \mathrm{H}$ and stretched samples is found, with the PM exhibiting a higher haze. Except for the LPCVD sample, all graphs shown in this section (Figs. 7 and 8) are normalized to their respective maxima. The graph of the LPCVD sample is normalized to the value of $23^{\circ}$ and $40.5^{\circ}$ at $600 \mathrm{~nm}$ and $1000 \mathrm{~nm}$, respectively.

Figures 7(a) and 7(b) show the AID for the small feature and large feature air samples, respectively. For both samples, the agreement of AID from PM and FDTD is nearly perfect; therefore validating the good agreement of the haze calculations as well.

Figures 8(a) and 8(b) show AID calculated from PM and FDTD for a high refractive index difference (large feature $\mu \mathrm{c}-\mathrm{Si}: \mathrm{H}$ sample) and a topography with steep flanks (stretched sample), respectively, for wavelengths of $600 \mathrm{~nm}$ and $1000 \mathrm{~nm}$. For the large feature $\mu \mathrm{c}-\mathrm{Si}: \mathrm{H}$ sample (Fig. 8(a)), the agreement between PM and FDTD is very good for both wavelengths, although haze calculations showed their maximum difference at $1000 \mathrm{~nm}$.

The stretched sample (Fig. 8(b)) shows larger differences between PM and FDTD. Agreement for a wavelength of $600 \mathrm{~nm}$ is very good with differences only at large angles. However, at a wavelength of $1000 \mathrm{~nm}$, the values exhibit


FIG. 7. Angular intensity distribution of 30 s (small feature sample, a) and 50s (large feature air sample, b) etched $\mathrm{ZnO}: \mathrm{Al}$ at a $\mathrm{ZnO}: \mathrm{Al} /$ air interface, calculated using PM and FDTD. AID is shown for $600 \mathrm{~nm}$ and $1000 \mathrm{~nm}$. All curves are normalized to their respective maxima. 



FIG. 8. Angular intensity distribution at a $\mathrm{ZnO}: \mathrm{Al} / \mu \mathrm{c}-\mathrm{Si}: \mathrm{H}$ interface (large feature $\mu \mathrm{c}-\mathrm{Si}: \mathrm{H}$ sample, a) and a numerically stretched sample at a $\mathrm{ZnO}: \mathrm{Al} /$ air interface (stretched sample, b). AID is shown for $600 \mathrm{~nm}$ and $1000 \mathrm{~nm}$. Both curves are normalized to their respective maxima.

some differences while general trends in the curve are still similar for both calculations. Especially the difference in the first point, which includes the specular portion of the transmitted light, explains the difference observed in haze calculations.

Looking at the LPCVD sample, shown in Fig. 9, we see an excellent agreement between PM and FDTD for the diffuse part of the AID (angle $>0^{\circ}$ ) except for large angles at $600 \mathrm{~nm}$. However, the PM calculation shows a stronger specular peak (angle $=0^{\circ}$ ), giving an explanation for the observed lower haze.

Overall agreement of AID calculations are very good, the AID of the large feature $\mu \mathrm{c}-\mathrm{Si}: \mathrm{H}$ and LPCVD samples shows a good but not perfect agreement of AID calculations, similar to the agreement of haze calculations.

While the angular resolution is identical for both approaches, and therefore the amount of information gained from both models is identical for AID and haze calculations, a higher angular resolution can resolve the features of the AID better, resulting in a better comparison between both models.

A higher angular resolution is only accessible through larger domains or a higher refractive index of the surrounding material, the first of which is not feasible due to the

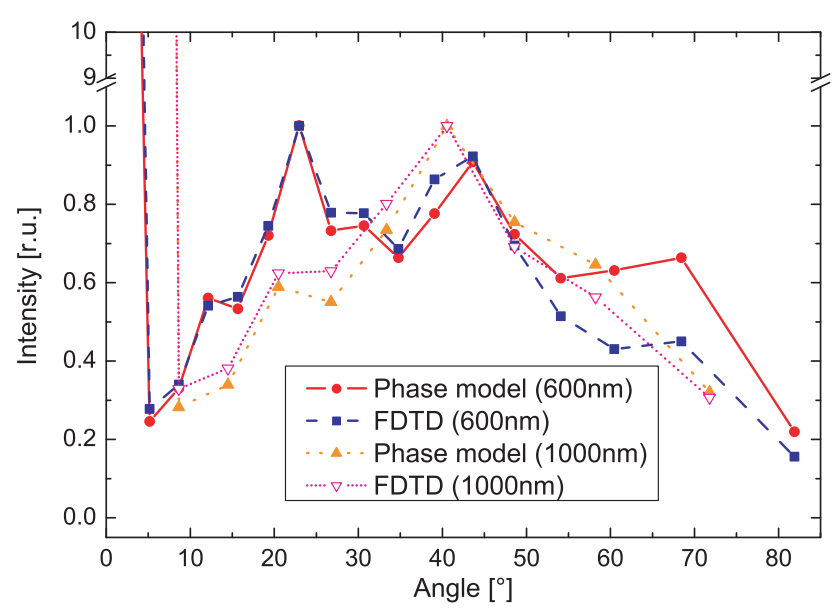

FIG. 9. Angular intensity distribution at a $\mathrm{ZnO}: \mathrm{Al} /$ air interface for the LPCVD sample. The curves at wavelengths of $600 \mathrm{~nm}$ and $1000 \mathrm{~nm}$ are normalized to the values at $23^{\circ}$ and $40.5^{\circ}$, respectively, with the PM showing a stronger peak at $0^{\circ}$.

computational complexity of FDTD simulations; the time needed for calculation increases linearly with increasing number of points, proportional to $x \cdot y \cdot z$, with $x, y, z$ being the number of points in the respective direction. Using a material with a higher refractive index as ambient is presented in the large feature $\mu \mathrm{c}-\mathrm{Si}: \mathrm{H}$ sample, which also results in a higher angular resolution.

\section{NEAR-FIELD EFFECTS}

Since it is easily accessible with the calculations used, the near-field effects (i.e., light scattered into evanescent modes) will also be checked for all samples at wavelengths of $600 \mathrm{~nm}$ and $1000 \mathrm{~nm}$ to examine the impact of the sharp features in the pupil function obtained by PM. Due to the nature of evanescent modes, these are only visible very close to the surface.

In thin-film solar cells, these evanescent modes are of great importance, ${ }^{24}$ since thicknesses and surface features are in a range in which near-field effects are present. In these models however, a slice perpendicular to the propagation direction is taken into account. For the PM, this is the only accessible plane. To have a direct comparison between FDTD and PM, the equivalent plane is considered in FDTD simulations.

Figures 10 (a) and 10(b) show the scattered light intensity as a function of the reciprocal lattice constant of the small feature sample and large feature air sample, respectively. The start of the evanescent modes, marked by vertical dashed and dotted lines for $1000 \mathrm{~nm}$ and $600 \mathrm{~nm}$, respectively, shows an extreme overestimation of the evanescent part by the PM. Results of both samples show a faster decline of evanescent modes in FDTD, resulting in one to two orders of magnitude offset while the shape of the curve is very similar for both calculations.

Figures 11(a) and 11(b) show evanescent modes of the large feature $\mu \mathrm{c}-\mathrm{Si}: \mathrm{H}$ sample and the stretched sample, respectively. The scattered light intensity exhibits the same behavior as for the samples shown in Fig. 10, but with an 

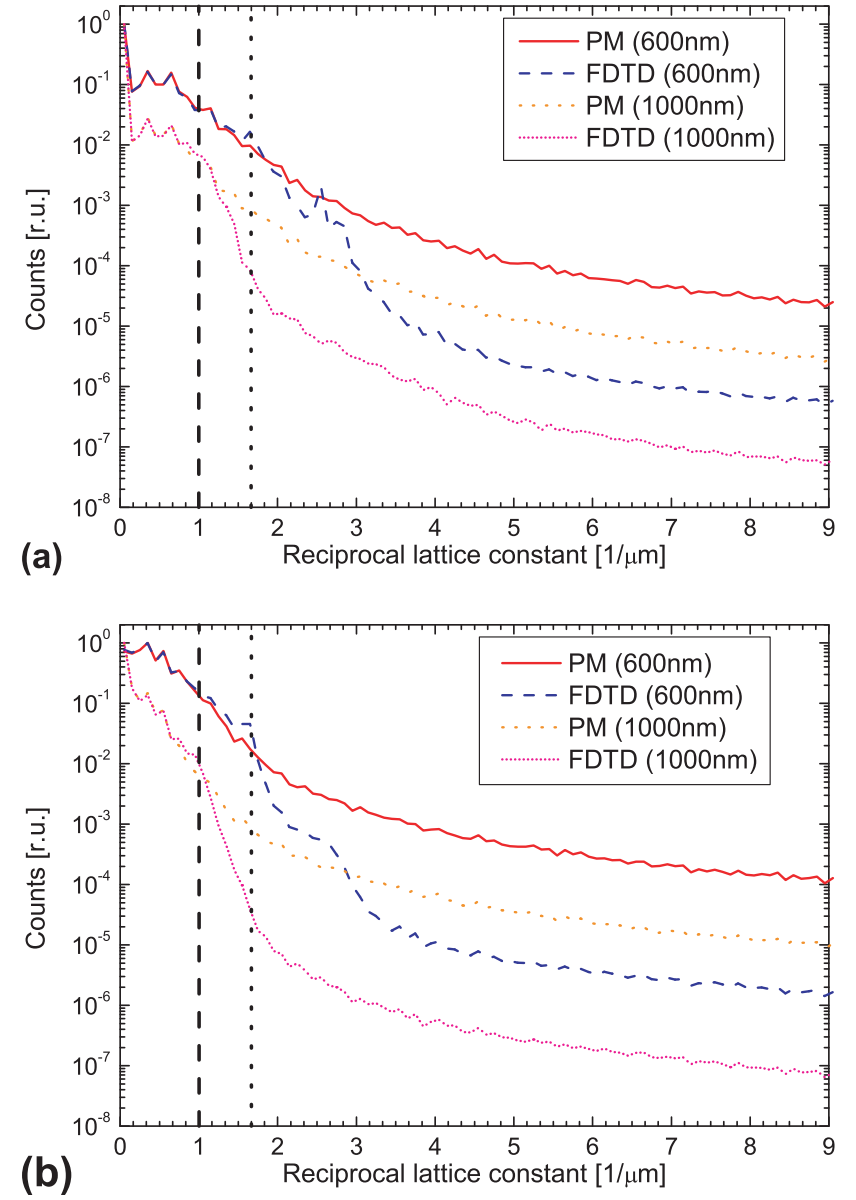

FIG. 10. Light scattered into evanescent modes for $\mathrm{ZnO}: \mathrm{Al} /$ air interfaces of the small feature sample (a) and large feature air sample (b). The dashed and dotted vertical lines represent the beginning of the evanescent modes for $1000 \mathrm{~nm}$ and $600 \mathrm{~nm}$, respectively.

even faster decline of the near-field modes for FDTD simulations (Fig. 11). This results in an even larger offset between PM and FDTD of at least 1.5 orders of magnitude. Looking at the light scattered into evanescent modes of the LPCVD sample (see Fig. 12), we see the same trends with an even larger difference of up to 3.5 orders of magnitude. Interestingly, both wavelengths of $600 \mathrm{~nm}$ and $1000 \mathrm{~nm}$ show nearly identical light scattered into evanescent modes when using the PM.

The reason for the difference between PM and FDTD lies in the nature of the PM. The evanescent modes decay exponentially with increasing distance from the interface. In FDTD simulation, this is taken into account, since it is a rigorous solution of Maxwell's equations. Looking at the pupil function obtained by PM, however, it is obvious that nearfield effects cannot be described correctly. Calculating the pupil function from a plane further away from the interface results in a constant phase shift for all points in respect to a plane at the maximum height of the topography. Using this new pupil function to calculate the intensity scattered into evanescent modes results in an identical distribution, so it does not depend on the distance from the surface, which contradicts the behavior of evanescent modes. This explains the difference between PM and FDTD.
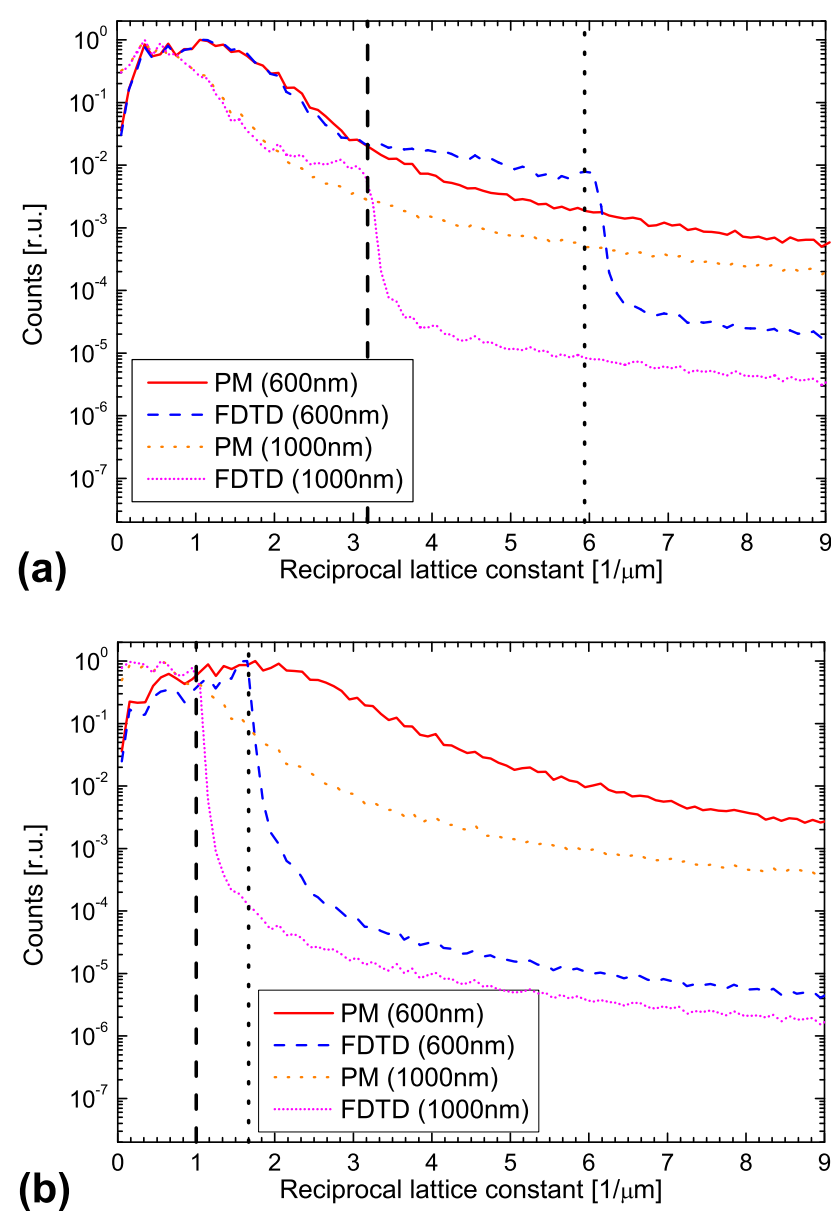

FIG. 11. Light intensity scattered into evanescent modes for the large feature $\mu \mathrm{c}-\mathrm{Si}: \mathrm{H}$ sample (ZnO:Al/ $\mu \mathrm{c}-\mathrm{Si}: \mathrm{H}$ interface, a) and stretched sample (b). The dashed and dotted vertical lines represent the beginning of the evanescent modes for $1000 \mathrm{~nm}$ and $600 \mathrm{~nm}$, respectively.

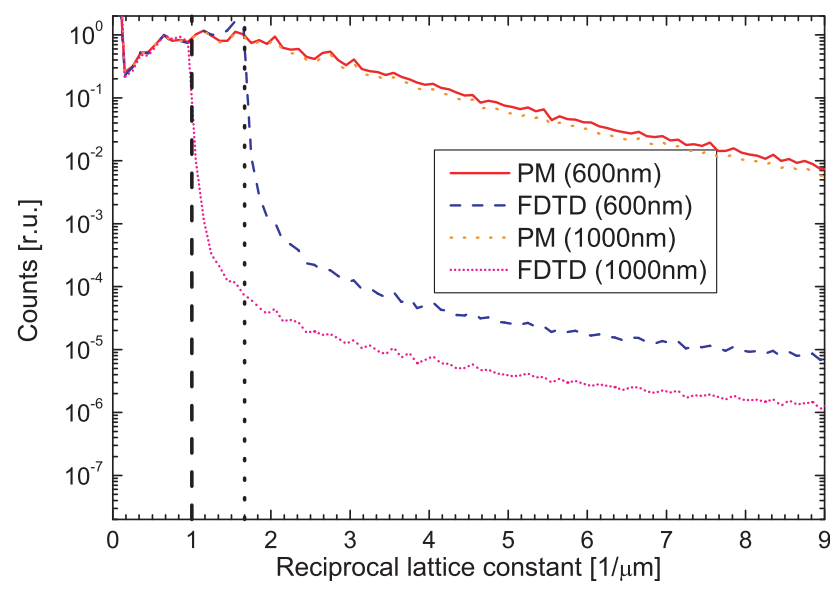

FIG. 12. Light intensity scattered into evanescent modes for LPCVD sample. The dashed and dotted vertical lines represent the beginning of the evanescent modes for $1000 \mathrm{~nm}$ and $600 \mathrm{~nm}$, respectively.

\section{CONCLUSION}

We have successfully compared the PM and FDTD simulations of various samples with varying textures, feature sizes, and refractive index differences. Haze calculations were made for a wide spectral range between $300 \mathrm{~nm}$ and 
$1300 \mathrm{~nm}$ with a resolution of $50 \mathrm{~nm}$. For all samples, the haze was very similar, with only minor differences for sharp features and/or a high refractive index change. AID calculations for both models were also in very good agreement with differences mainly due to the limited angular resolution due to the small domain size. Investigation of the evanescent modes exhibited a strong over-estimation by the PM, the reason of which being the constant phase shift obtained by the PM when looking at a plane further away from the top of the surface. Due to the analysis used to obtain the scattered light (both as AID and scattered into evanescent modes), this distance has no influence at all on the scattering behavior, therefore light scattered into evanescent modes cannot be described correctly, since these modes decay exponentially with the distance from the surface.

In conclusion, the phase model proved a very powerful tool to estimate the scattering properties of different surface types for different material combinations. This can be effectively used to see if a very time consuming FDTD simulation of a given system is justified.

However, a rigorous solution of Maxwell's equations is necessary for the optical modeling of a solar cell device. First of all, the PM is limited to a single interface. But there are several interfaces in a typical solar cell which lead to different and/or additional scattering as well as interferences. With some of the layers in these systems being only a few nanometers thick, near-field effects are also important. And finally, there is absorption in each layer, which is crucial for the solar cell device.

\section{ACKNOWLEDGMENTS}

We would like to thank M. Schulte for real-world structures and measurements for comparison and inspiration, as well as U. Paetzold for inspiring and productive discussion. Additionally, we thank Professor Dr. U. Rau for continuous support. We also appreciate the ability to use the JUROPA HPC cluster at JSC, FZ Jülich for FDTD simulations via the VSR project JIPV00, as well as the partial financial support by the BMBF, project PhoNa (03IS101F) and the DFG, project Nanosun (PAK88), and project LIST(0325299A) of the BMU.

${ }^{1}$ M. Berginski, J. Hüpkes, M. Schulte, G. Schöpe, H. Stiebig, B. Rech, and M. Wuttig, J. Appl. Phys. 101, 074903 (2007).

${ }^{2}$ D. Zhou and R. Biswas, J. Appl. Phys. 103, 093102 (2008).

${ }^{3}$ V. E. Ferry, M. A. Verschuuren, H. B. T. Li, E. Verhagen, R. J. Walters, R. E. I. Schropp, H. A. Atwater, and A. Polman, Opt. Express 18, A237A245 (2010).

${ }^{4}$ P. N. Saeta, E. Vivian, D. Pacifici, J. N. Munday, and H. A. Atwater, Opt. Express 17, 20975-20990 (2009).

${ }^{5}$ U. W. Paetzold, E. Moulin, D. Michaelis, W. Böttler, C. Wächter, V. Hagemann, M. Meier, R. Carius, and U. Rau, Appl. Phys. Lett. 99, 181105 (2011).

${ }^{6}$ H. W. Deckman, C. R. Wronski, H. Witzke, and E. Yablonovitch, Appl. Phys. Lett. 42, 968 (1983).

${ }^{7}$ C. Haase and H. Stiebig, Appl. Phys. Lett. 91, 061116 (2007).

${ }^{8}$ C. Rockstuhl, S. Fahr, K. Bittkau, T. Beckers, R. Carius, F.-J. Haug, T. Söderström, C. Ballif, and F. Lederer, Opt. Express. 18, A335-A341 (2010).

${ }^{9}$ H. Schade and Z. E. Smith, Appl. Opt. 24, 3221 (1985).

${ }^{10} \mathrm{G}$. Tao, PhD thesis, Technical University Delft, 1994.

${ }^{11}$ R. Brendel, Prog. Photovoltaics 3, 25 (1995).

${ }^{12}$ S. Fahr, C. Rockstuhl, and F. Lederer, Appl. Phys. Lett. 92, 171114 (2008).

${ }^{13}$ E. Yablonovitch and G. D. Cody, IEEE Trans. Electron devices ED-29, 300-305 (1982).

${ }^{14}$ E. Yablonovitch, Opt. Soc. Am. 72, 899-907 (1982).

${ }^{15}$ C. K. Carniglia, Opt. Eng. 18, 182104 (1979).

${ }^{16}$ J. Krč, M. Zeman, O. Kluth, F. Smole, and M. Topic, Thin Solid Films 426, 296 (2003).

${ }^{17}$ D. Dominé, F.J.-Haug, C. Battaglia, and C. Ballif, J. Appl. Phys. 107, 044504 (2010).

${ }^{18}$ K. Jäger, M. Fischer, R. A. C. M. M. van Swaaij, and M. Zeman, J. Appl. Phys. 111, 083108 (2012).

${ }^{19}$ A. Taflove and S. C. Hagness, Computational Electrodynamics: The Finite-Difference Time-Domain Method (Artech: Norwood, MA, 2000).

${ }^{20}$ M. Schulte, K. Bittkau, K. Jäger, M. Ermes, M. Zeeman, and B. E. Pieters, Appl. Phys. Lett. 99, 111107 (2011).

${ }^{21}$ C. Rockstuhl, S. Fahr, F. Lederer, F.-J. Haug, T. Söderström, S. Nicolay, M. Despeisse, and C. Ballif, Appl. Phys. Lett. 98, 051102 (2011).

${ }^{22}$ C. Battaglia, J. Escarré, K. Södertröm, M. Boccard, and C. Ballif, Energy Procedia 15, 206-211 (2012).

${ }^{23}$ A. F. Oskooi, D. Roundy, M. Ibanescu, P. Bermel, J. D. Joannopoulos, and S. G. Johnson, Comp. Phys. Comm. 181, 687-702 (2010).

${ }^{24}$ K. Bittkau and T. Beckers, Phys. Status Solidi A 207, 661-666 (2010). 